\title{
Constraining anomaly mediated supersymmetry breaking framework via ongoing muon $g-2$ experiment at Brookhaven
}

\author{
Utpal Chattopadhyay* \\ Department of Theoretical Physics, Tata Institute of Fundamental Research, Homi Bhabha Road, Mumbai 400 005, India \\ Dilip Kumar Ghosh ${ }^{\dagger}$ \\ CERN, Theory Division, CH-1211 Geneva 23, Switzerland \\ Sourov Roy \\ Department of Theoretical Physics, Tata Institute of Fundamental Research, Homi Bhabha Road, Mumbai 400 005, India
}

(Received 6 June 2000; published 16 October 2000)

\begin{abstract}
The ongoing high precision E821 Brookhaven National Laboratory experiment on muon $g-2$ is promising to probe a theory involving supersymmetry. We have studied the constraints on the minimal anomaly mediated supersymmetry breaking (AMSB) model using the current data of muon $g-2$ from Brookhaven. A scenario of seeing no deviation from the standard model is also considered, within a $2 \sigma$ limit of the combined error from the standard model result and the Brookhaven predicted uncertainty level. The resulting constraint is found to be complementary to what one obtains from $b \rightarrow s+\gamma$ bounds within the AMSB scenario, since only a definite sign of $\mu$ is effectively probed via $b \rightarrow s+\gamma$. A few relevant generic features of the model are also described for disallowed regions of parameter space.

PACS number(s): 12.60.Jv, 04.65.+e, 13.40.Em, 14.60.Ef
\end{abstract}

\section{INTRODUCTION}

The search for supersymmetry (SUSY) in high energy physics relies both on high energy colliders as well as on experiments based on perturbative corrections to various experimentally measurable quantities. Traditionally, the measurement of an electron's anomalous magnetic moment has been highly effective in verifying the prediction of quantum electrodynamics (QED) to a very high order. Probing beyond the standard model physics with supersymmetry is seen to be possible with a precision $(g-2)$ measurement of the muon. The ongoing muon $(g-2)$ measurement E821 [1] at Brookhaven National Laboratory (BNL), designed to verify the results of standard model (SM) electroweak corrections, has already provided a more accurate result than the previous CERN experiment [2], by a factor of 2 or so. With improved design and state of the art technology, it is expected that within a few years from now, the accuracy of the BNL result will be increased by a factor of 20 , or even more, compared to the same of the previous CERN measurement.

Supersymmetric electroweak corrections to muon ( $g$ -2) can be as large as the SM electroweak correction, and this fact has been seen in a number of references ranging from the minimal supersymmetric standard model (MSSM) $[3,4]$, supergravity based models $[5,6]$, and gauge mediated supersymmetry breaking scenarios [7]. In the recent past, considerable interest has been seen in a different type of SUSY breaking mechanism, other than supergravity [8] and

\footnotetext{
*Email address: utpal@theory.tifr.res.in

${ }^{\dagger}$ On leave of absence from Department of Theoretical Physics,

TIFR, Mumbai, India. Email address: Dilip.Ghosh@cern.ch

ॠEmail address: sourov@theory.tifr.res.in
}

gauge mediated SUSY breaking [9], which, at its dominating scenario, is generically known as anomaly mediated supersymmetry breaking (AMSB) [10-12]. This effect originates from the existence of a super Weyl anomaly [10] while considering SUSY breaking. As we will discuss later, the problem of the resulting tachyonic sleptons, which arises within the AMSB sparticle spectrum, is avoided in the minimal definition [13-18] of the model, via adding a common scalar mass $m_{0}$ with all the scalars of the theory, at a given scale.

Large SUSY contributions $a_{\mu}^{\mathrm{SUSY}} \equiv \frac{1}{2}(g-2)_{\mu}^{\mathrm{SUSY}}$ in the minimal AMSB framework have already been seen in Ref. [13], in which the authors discussed in detail a broad range of interesting phenomenological implications involving colliders, as well as various low energy signatures within the model, in addition to showing that the minimal model may remain natural even for a superheavy $m_{0}$. In this work, we will analyze the constraint coming from $a_{\mu}^{\text {SUSY }}$ with regard to the high precision Brookhaven experiment, within the minimal AMSB framework. Considering $a_{\mu}^{\mathrm{expt}}-a_{\mu}^{\mathrm{SM}}=a_{\mu}^{\mathrm{SUSY}}$ and taking into account the associated error limits in quadratures, we will constrain the SUSY parameter space of the model.

Our work will be organized as follows. In Sec. II, we will discuss the SM result for $a_{\mu}$ for its different parts of contributions. We will see the existence of a large error associated with the lowest order hadronic vacuum polarization contribution and sources of its possible improvement in the near future, via low energy $e^{+} e^{-} \rightarrow$ hadron data from various experiments. We will describe the AMSB framework and the necessity of defining a minimal scenario in Sec. III. In Sec. IV, we will use Ref. [5] for the result of $a_{\mu}^{\text {SUSY }}$, where the analysis was performed in the minimal supergravity (MSUGRA) model to see the constraint from the high precision BNL experiment. We will analyze the constraint from $a_{\mu}^{\text {SUSY }}$ on the parameter space of the minimal AMSB model, 
TABLE I. Contributions to $a_{\mu}^{\mathrm{SM}}$ (in units of $10^{-10}$ ).

\begin{tabular}{lc}
\hline \multicolumn{1}{c}{ Nature of contribution } & Value \\
\hline QED to $O(\alpha / \pi)^{5}[19]\left(a_{\mu}^{\mathrm{QED}}\right)$ & $11658470.6(0.3)$ \\
\hline Hadronic vac. polarization to $O(\alpha / \pi)^{2}\left(a_{\mu}^{\text {had1 }}\right)[20]$ & $692.4(6.2)$ \\
Hadronic vac. polarization to $O(\alpha / \pi)^{3}[21]$ & $-10.1(0.6)$ \\
Light-by-light hadronic amplitude $[22]$ & $-7.92(1.54)$ \\
\hline Total hadronic $\left(a_{\mu}^{\text {hadronic }}\right)$ & $677.1(6.5)$ \\
\hline Total electroweak up to two loops $\left(a_{\mu}^{\mathrm{EW}}\right)$ & $15.1(0.4)$ \\
\hline$a_{\mu}^{\mathrm{SM}}$ & $11659160.1(6.5)$ \\
\hline \hline
\end{tabular}

due to the present SM and experimental results of $a_{\mu}$. We will also investigate the potential for constraining the minimal AMSB model using the predicted level of the uncertainty of the E821 BNL experiment. We will do so in the minimal no-deviation scenario, which here means seeing no disagreement from the SM result within the experimental and the theoretical uncertainties, once the measurement is complete at the desired level of accuracy. We will also quote the result of the $b \rightarrow s+\gamma$ constraint for both signs of $\mu$ and the positive role of an $a_{\mu}^{\text {SUSY }}$ analysis in this regard. In Sec. $\mathrm{V}$, we will comment on the disallowed regions which appear

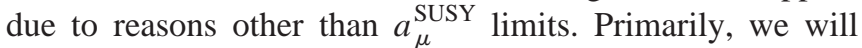
examine the disallowed regions of parameters space due to the combined effect of the scale invariant part of the scalar mass relations of sleptons with gauge and Yukawa couplings within the minimal AMSB model and the nature of the associated renormalization group evolutions. We will also see the effect of SUSY-QCD corrections to the bottom-quark mass on the minimal AMSB spectra, a large $\tan \beta$ effect, which also has specific features within AMSB models. These regions, when combined with $a_{\mu}^{\text {SUSY }}$ eliminated parameter space, provide simpler and definite predictions for the lower bounds of the masses of the relevant supersymmetric particles, as well as of the input parameters of the model.

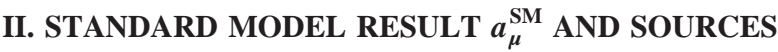 OF UNCERTAINTY}

The standard model result for $a_{\mu}$ is

$$
\begin{aligned}
\frac{1}{2}(g-2){ }_{\mu}^{\mathrm{SM}} & =a_{\mu}^{\mathrm{SM}} \\
& =11659162.8(6.5) \times 10^{-10} \\
& \equiv(11659162.8 \pm 6.5) \times 10^{-10} .
\end{aligned}
$$

In contrast, the latest data from the ongoing E821 BNL experiment [1] amounts to

$$
a_{\mu}^{\mathrm{expt}}=11659210(46) \times 10^{-10} .
$$

The uncertainty amount is expected to be reduced to $\delta a_{\mu}^{\mathrm{BNL}}$ $\lesssim 4 \times 10^{-10}$.

The SM result is broken up into

$$
a_{\mu}^{\mathrm{SM}}=a_{\mu}^{\mathrm{QED}}+a_{\mu}^{\text {hadronic }}+a_{\mu}^{\mathrm{EW}} .
$$

Here $a_{\mu}^{\text {QED }}$ is the pure QED contribution computed up to five loops in electromagnetic coupling. The quantity $a_{\mu}^{\text {hadronic }}$ refers to the total hadronic contribution including the lowest order and the next to lowest order hadronic vacuum polarizations and the light-by-light hadronic contribution. The electroweak part $a_{\mu}^{\mathrm{EW}}$ is the SM electroweak contribution up to two loops. The amounts from the individual parts with corresponding references are summarized in Table I.

Within $a_{\mu}^{\text {hadronic }}$, the contribution $a_{\mu}^{\text {had } 1}$ arising from the $\alpha^{2}$ level of hadronic vacuum polarization diagram is the least accurate quantity, and its uncertainty is almost the same as the overall error of $a_{\mu}^{\mathrm{SM}}$. Hence an accurate determination of $a_{\mu}^{\text {had } 1}$ will be increasingly important for compatibility with the high precision measurements at BNL, which have an expected level of uncertainty of $\delta a_{\mu}^{\mathrm{BNL}} \lesssim 4 \times 10^{-10}$. However, the present uncertainty in $a_{\mu}^{\mathrm{SM}}$ is quite small compared to the experimental uncertainty level, a situation which is going to be changed within a few years. The largest hadronic contribution $a_{\mu}^{h a d 1}$ is obtained from the total Born cross section (lowest order in QED) for hadron production in $e^{+} e^{-}$ annihilation, a result found via a dispersion theory and optical theorem [23]. Accurate low energy $e^{+} e^{-} \rightarrow$ hadron data hence become necessary to lower the uncertainty level. Measurements of low energy hadron production cross sections in BES, CMD-II, and DAФNE [24] will significantly improve the result of $a_{\mu}^{\mathrm{SM}}$. In the recent past, the authors of Ref. [20] used ALEPH data from hadronic $\tau$ decay and QCD sum-rule techniques to evaluate $a_{\mu}^{\text {had } 1}$ and this improved the hadronic error estimate very significantly. Our analysis uses this result. Further prospects for improving the result of $a_{\mu}^{h a d 1}$, as well as a critical evaluation of the above estimate may be seen in Ref. [25].

\section{ANOMALY MEDIATED SUPERSYMMETRY BREAKING IN THE MINIMAL SCENARIO}

In a supersymmetric theory, additionally, soft SUSY breaking parameters are also contributed via a super Weyl anomaly, which is a generic feature if SUSY is broken in a supergravity framework. In the anomaly mediated supersymmetry breaking scenario, the super Weyl anomaly contribu- 
tions dominate, because the SUSY breaking sector and the visible sector reside in different parallel three-branes [10] in a string theoretical perspective, and there are no tree-level couplings between the two branes. The form of soft parameters thus generated is renormalization group (RG) invariant, and at any desired scale, the soft parameters are determined by the appropriate gauge and Yukawa couplings for the same scale. This is particularly interesting to avoid a SUSY flavor problem, because the scale invariance of soft parameters which is provided by special RG trajectories eliminates the effect of any flavor violating unknown physics possibly existing at a higher scale.

In spite of many desirable features of anomaly mediation, within the framework of the MSSM, one finds that sleptons become tachyonic. In the minimal AMSB model such tachyonic sleptons are avoided by introducing an additional common mass parameter $m_{0}$ for all the scalars of the theory. But this obviously violates the scale invariance of the model, whereas preserving the same would be desirable in regard to the flavor changing neutral current (FCNC) constraint. Tachyonic sleptons are avoided differently in nonminimal AMSB models [26], which have appropriate scale invariant scalar mass combinations within RG evolutions, but these are outside the scope of our present work. However, via the existence of a focus point [27] of the renormalization group equation (RGE) of $m_{H_{u}}^{2}$, the minimal model allows the possibility of multi-TeV squarks and sleptons without increasing the fine-tuning measure [13], and this is an important feature to address many phenomenological issues.

Scalar masses are hence determined via renormalization group equations of the MSSM starting from their respective values at the unification scale $\left(M_{G} \sim 1.5-2.0 \times 10^{16} \mathrm{GeV}\right)$ and leading up to the electroweak scale $M_{Z}$, the scale for mass of the $Z$ boson. However, for the first two generations of scalars, because of the negligible first two generation Yukawa couplings, the effect translates to having simply an overall additive constant $m_{0}$ at $M_{G}$, which would have minimal changes due to $R G$ evolutions.

In this analysis, the evolutions of scalar masses, gauge, and Yukawa couplings are computed at the two-loop level of the RGE [28], and trilinear couplings are evolved via the one-loop level of the same. Unification of gauge couplings is incorporated with having $\alpha_{3}\left(M_{Z}\right) \sim 0.118$. For Higgsino mixing, $\mu^{2}$ is computed radiatively via an electroweak symmetry breaking condition at the complete one-loop level of the effective potential [29], while optimally choosing a renormalization scale $Q=\sqrt{\left(m_{t_{1}} m_{t_{2}}\right)}$ for minimization. The analysis also includes a supersymmetric QCD correction to the bottom-quark mass [30], which is considerable for large $\tan \beta$ regions also having its important features in AMSB scenarios.

With a high degree of predictivity the model is described by the following parameters: the gravitino mass $m_{3 / 2}$, the common scalar mass parameter $m_{0}$, the ratio of Higgs vacuum expectation values $\tan \beta$, and $\operatorname{sgn}(\mu)$. Following Ref. [31], having the same sign conventions for $\mu$ and $A$ parameters in this work, we see that the masses are given via the couplings as follows.
Gauginos:

$$
\begin{aligned}
& \tilde{m}_{1}=\frac{33}{5} \frac{g_{1}^{2}}{16 \pi^{2}} m_{3 / 2}, \quad \tilde{m}_{2}=\frac{g_{2}^{2}}{16 \pi^{2}} m_{3 / 2}, \\
& \tilde{m}_{3}=-3 \frac{g_{3}^{2}}{16 \pi^{2}} m_{3 / 2} .
\end{aligned}
$$

Higgs and third generation scalars:

$$
\tilde{m}_{i}^{2}=C_{i} \frac{m_{3 / 2}^{2}}{\left(16 \pi^{2}\right)^{2}}+m_{0}^{2},
$$

where $i \equiv\left(Q, U, D, L, E, H_{u}, H_{d}\right)$, with $C_{i}$ 's being given as

$$
\begin{aligned}
& C_{Q}=-\frac{11}{50} g_{1}^{4}-\frac{3}{2} g_{2}^{4}+8 g_{3}^{4}+h_{t} \hat{\beta}_{h_{t}}+h_{b} \hat{\beta}_{h_{b}}, \\
& C_{U}=-\frac{88}{25} g_{1}^{4}+8 g_{3}^{4}+2 h_{t} \hat{\beta}_{h_{t}} \\
& C_{D}=-\frac{22}{25} g_{1}^{4}+8 g_{3}^{4}+2 h_{b} \hat{\beta}_{h_{b}}, \\
& C_{L}=-\frac{99}{50} g_{1}^{4}-\frac{3}{2} g_{2}^{4}+h_{\tau} \hat{\beta}_{h_{\tau}}, \\
& C_{E}=-\frac{198}{25} g_{1}^{4}+2 h_{\tau} \hat{\beta}_{h_{\tau}}, \\
& C_{H_{u}}=-\frac{99}{50} g_{1}^{4}-\frac{3}{2} g_{2}^{4}+3 h_{t} \hat{\beta}_{h_{t}},
\end{aligned}
$$

and

$$
C_{H_{d}}=-\frac{99}{50} g_{1}^{4}-\frac{3}{2} g_{2}^{4}+3 h_{b} \hat{\beta}_{h_{b}}+h_{\tau} \hat{\beta}_{h_{\tau}}
$$

Here, $Q$ and $L$ are the superpartners of quark and lepton doublet fields, respectively. The superpartners for singlet quark fields for up and down type are $U$ and $D$, and the same for singlet lepton is $E$.

Trilinear couplings:

$A_{t}=\frac{\hat{\beta}_{h_{t}}}{h_{t}} \frac{m_{3 / 2}}{16 \pi^{2}}, \quad A_{b}=\frac{\hat{\beta}_{h_{b}}}{h_{b}} \frac{m_{3 / 2}}{16 \pi^{2}}, \quad$ and $\quad A_{\tau}=\frac{\hat{\beta}_{h_{\tau}}}{h_{\tau}} \frac{m_{3 / 2}}{16 \pi^{2}}$,

where $\hat{\beta}$ 's are defined by

$$
\begin{aligned}
& \hat{\beta}_{h_{t}}=h_{t}\left(-\frac{13}{15} g_{1}^{2}-3 g_{2}^{2}-\frac{16}{3} g_{3}^{2}+6 h_{t}^{2}+h_{b}^{2}\right), \\
& \hat{\beta}_{h_{b}}=h_{b}\left(-\frac{7}{15} g_{1}^{2}-3 g_{2}^{2}-\frac{16}{3} g_{3}^{2}+h_{t}^{2}+6 h_{b}^{2}+h_{\tau}^{2}\right),
\end{aligned}
$$


and

$$
\hat{\beta}_{h_{\tau}}=h_{\tau}\left(-\frac{9}{5} g_{1}^{2}-3 g_{2}^{2}+3 h_{b}^{2}+4 h_{\tau}^{2}\right) .
$$

The quantities for the first two generations can be obtained similarly by considering appropriate Yukawa couplings, which, however, are neglected in our analysis. We note that, Eq. (4) and Eq. (6) are scale invariant. Hence, having no intrinsic $\mathrm{RG}$ evolutions of their own, the masses and the trilinear couplings may be computed at any scale once the appropriate gauge and Yukawa couplings are known. However, because of the addition of the $m_{0}^{2}$ term, which rescues sleptons from being tachyonic, Eq. (5) is not scale invariant. Here the scale for obtaining the mass values of Eq. (5) is chosen as $M_{G}$. Thereafter, RG evolutions of soft scalar parameters and use of the electroweak radiative breaking condition at the complete one-loop level produce the sparticle mass spectra. One of the important features of the minimal AMSB model is that the resulting SU(2) gaugino mass $\tilde{m}_{2}$ is quite smaller than $\tilde{m}_{1}$ as well as $|\mu|$. Here, we have also incorporated the non-negligible next to leading order (NLO) corrections [14] for gaugino masses. As a result, the lighter chargino $\tilde{\chi}_{1}^{ \pm}$and lightest neutralino $\tilde{\chi}_{1}^{0}$ are $W$-ino dominated; indeed they are almost degenerate, with the latter becoming the lightest supersymmetric particle (LSP). This has interesting phenomenological aspects, like what is seen most recently in Ref. [17], where a definite signal in a linear $e^{+} e^{-}$ collider could be predicted as a possible minimal AMSB signature. Compared to other SUSY scenarios where the lightest neutralino has a distinctly smaller mass, here this similar mass value of $m \tilde{\chi}_{1}^{ \pm}$and $m \tilde{\chi}_{1}^{0}$ effectively decreases $\left|a_{\mu}^{\text {SUSY }}\right|$, although a weakly contributing effect. Another striking result of the minimal AMSB model is the strong mass degeneracy between left and right sleptons. Consequently, the third and the second generation $L-R$ mixing angles become significantly larger, going up to the maximal limit for a large $\tan \beta$. We will see the strong effect of large smuon $L-R$ mixing on the neutralino loop contributions of $a_{\mu}^{\mathrm{SUSY}}$ in Sec. IV.

\section{RESULTS}

The diagrams to compute the supersymmetric contributions to muon $(g-2)$, as shown in Figs. 1(a) and 1(b), are divided into the chargino-sneutrino loop and the neutralinosmuon loop. We only quote the chargino part of the result here, which dominates in $a_{\mu}^{\text {SUSY }}$. The neutralino contribution may be seen in Refs. [3,5]. ${ }^{1}$ Separating the chargino contributions into chirality diagonal and nondiagonal parts we have

\footnotetext{
${ }^{1}$ The most general result of SUSY electroweak contribution to muon $(g-2)$ in the MSSM, where $C P$ violating phases are considered, can be seen in Ref. [3].
}

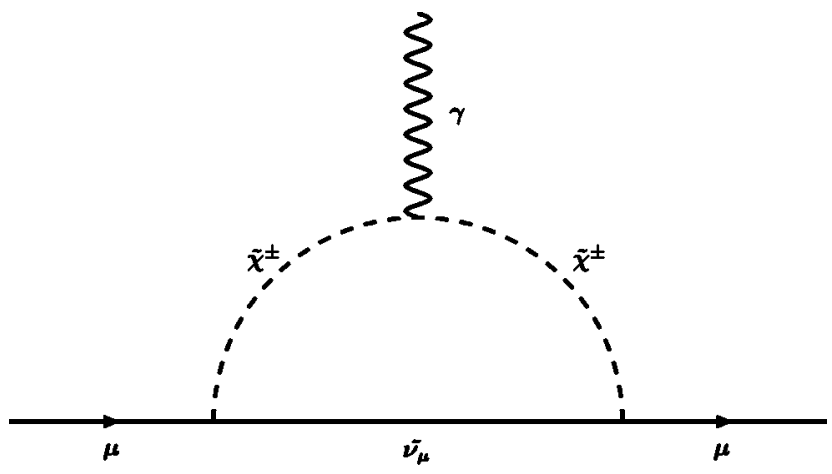

(a)

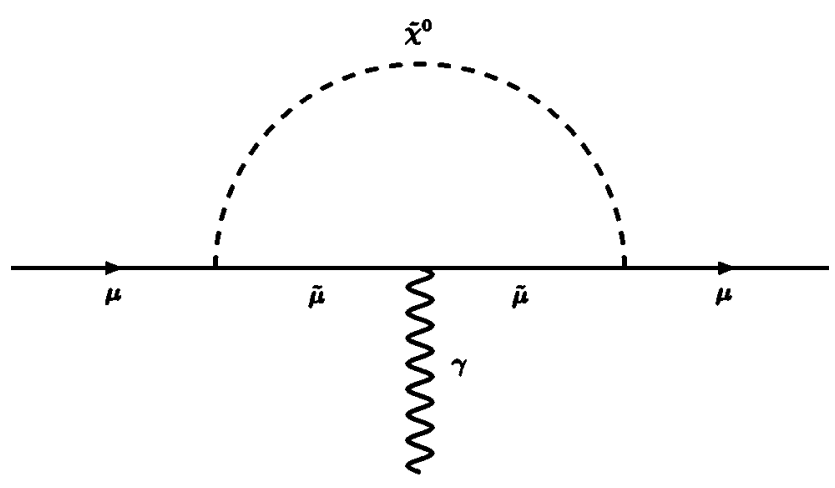

(b)

FIG. 1. Feynman diagrams contributing to $a_{\mu}^{\text {SUSY }}$ (a) for the chargino-sneutrino loop, (b) for the neutralino-smuon loop.

$$
a_{\mu}^{\mathrm{SUSY} \chi^{ \pm}}=a_{\mu}^{\mathrm{SUSY} \chi^{ \pm}}(\text {nondiag })+a_{\mu}^{\mathrm{SUSY}_{\chi^{ \pm}}}(\operatorname{diag}),
$$

where

$$
\begin{aligned}
a_{\mu}^{\operatorname{SUSY}_{\chi^{ \pm}}(\text {nondiag })=} & \frac{m_{\mu}^{2} \alpha_{e m}}{4 \sqrt{2} \pi m_{W} \sin ^{2} \theta_{W} \cos \beta} \\
& \times \sum_{i=1}^{2} \frac{1}{m_{\chi_{i}^{ \pm}}}\left(U_{i 2} V_{i 1}\right) F\left(\frac{m_{\tilde{\nu}_{\mu}}^{2}}{m_{\chi_{i}^{ \pm}}^{2}}\right)
\end{aligned}
$$

and

$$
\begin{aligned}
a_{\mu}^{\operatorname{SUSY}_{\chi^{ \pm}}}(\operatorname{diag})= & \frac{m_{\mu}^{2} \alpha_{e m}}{24 \pi \sin ^{2} \theta_{W}} \sum_{i=1}^{2} \frac{1}{m_{\chi_{i}^{ \pm}}^{2}} \\
& \times\left(\frac{m_{\mu}^{2}}{2 m_{W}^{2} \cos ^{2} \beta} U_{i 2}^{2}+V_{i 1}^{2}\right) G\left(\frac{m_{\tilde{\nu}_{\mu}}^{2}}{m_{\chi_{i}^{ \pm}}^{2}}\right) .
\end{aligned}
$$

Here, in general, $U$ and $V$ are unitary $2 \times 2$ matrices, which diagonalize the chargino mass matrix $M_{\tilde{\chi}^{ \pm}}$as shown below, via a bi-unitary transformation, $U^{*} M_{\tilde{\chi}^{ \pm}} V^{-1}$ $=\operatorname{diag}\left(m_{\tilde{\chi}_{1}^{ \pm}}^{ \pm}, m_{\chi_{2}^{ \pm}}^{ \pm}\right)$: 

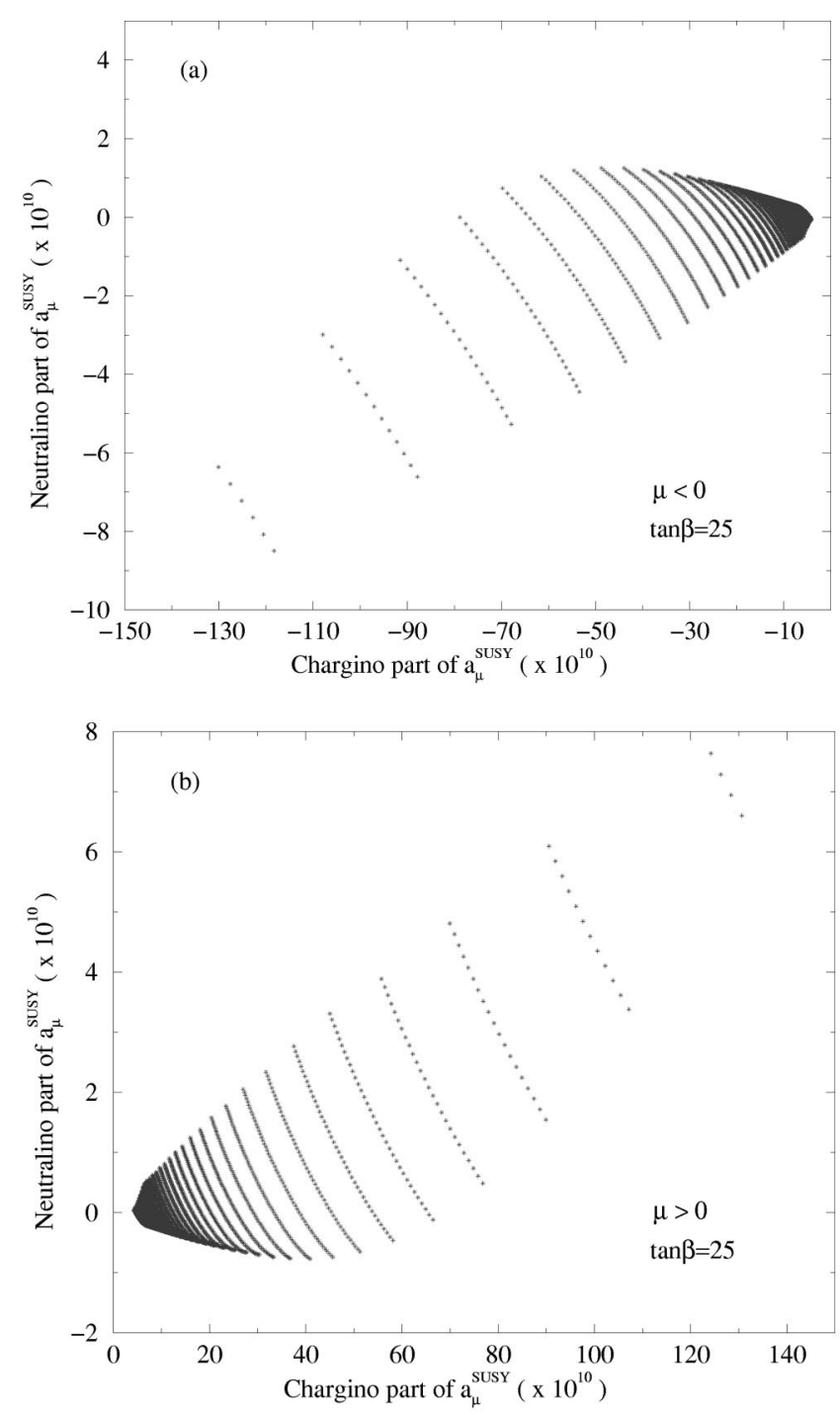

FIG. 2. Relative contributions to muon $a_{\mu}^{\text {SUSY }}$ for (a) $\mu<0$ and (b) $\mu>0$ from chargino-sneutrino and neutralino-smuon loops when $\tan \beta=25, m_{0} \leqslant 1000 \mathrm{GeV}$, and $m_{3 / 2} \leqslant 100 \mathrm{TeV}$.

$$
M_{\tilde{\chi}^{ \pm}}=\left(\begin{array}{cc}
\tilde{m}_{2} & \sqrt{2} m_{W} \sin \beta \\
\sqrt{2} m_{W} \cos \beta & \mu
\end{array}\right) .
$$

With $M_{\tilde{\chi}^{ \pm}}$being real $U$ and $V$ are orthogonal matrices. The functions $F(x)$ and $G(x)$ arising from loop integrations are given by $F(x)=\left(3 x^{2}-4 x+1-2 x^{2} \ln x\right) /(x-1)^{3}$ and $G(x)=\left(2 x^{3}+3 x^{2}-6 x+1-6 x^{2} \ln x\right) /(x-1)^{4}$.

Using the complete $a_{\mu}^{\text {SUSY }}$ result for numerical computations, Figs. 2(a) and 2(b) show the dominance of chargino contributions over the neutralino parts. Here, the two types of contributions are plotted along the axes for $\tan \beta=25$, when $m_{3 / 2}$ and $m_{0}$ are varied over a broad range of values $\left(m_{3 / 2}<100 \mathrm{TeV}\right.$ and $\left.m_{0}<1 \mathrm{TeV}\right)$. It is indeed the chirality nondiagonal term involving the lighter chargino and sneutrino part which dominates over the other contributions in $a_{\mu}^{\text {SUSY }}$. Because of the same reason, as explained further in a similar MSUGRA analysis of Ref. [5], there is a definite sign dependence between $a_{\mu}^{\text {SUSY }}$ and $\mu$, namely, $a_{\mu}^{\text {SUSY }}>0$ for $\mu>0$, and $a_{\mu}^{\text {SUSY }}<0$ for $\mu<0$, an important result for $a_{\mu}^{\text {SUSY }}$. Thus, the lighter chargino mass $\left(m_{\tilde{\chi}_{1}^{ \pm}}\right)$has a significant role in $a_{\mu}^{\mathrm{SUSY}}$. On the other hand, for a given $m_{\tilde{\chi}_{1}^{ \pm}}$value, a heavier $m_{\tilde{v}_{\mu}}$ decreases $\left|a_{\mu}^{\mathrm{SUSY}}\right|$. Because of the presence of Yukawa coupling $(\sim 1 / \cos \beta)$ within the chirality nondiagonal terms for both the chargino [see Eq. (8)] and neutralino results, we see that $\left|a_{\mu}^{\text {SUSY }}\right|$ is almost proportional to $\tan \beta$.

The special signature of AMSB due to an extremely large smuon $L-R$ mixing angle, as mentioned before, affects the neutralino results to diminish strongly via partial cancellation between the terms. The particular neutralino term (see Ref. [5]), which involves smuon mixing, becomes almost comparable to the significantly contributing chirality nondiagonal neutralino term associated with Yukawa coupling. Both terms depend on $\tan \beta$, as well as on the sign of $\mu$. A detail numerical investigation shows that the two terms always come in opposite signs, giving a large cancellation within the neutralino result. On a relative scale, we have seen that, for a naturalness [32] favored region of SUSY spectra with a given $\tan \beta$, the ratio of neutralino to chargino contributions within $a_{\mu}^{\text {SUSY }}$ in minimal AMSB is typically smaller by $50 \%$ or so, compared to the same within a similar natural MSUGRA spectra.

\section{A. Constraints from present values of $a_{\mu}^{\text {expt }}$ and $a_{\mu}^{\mathrm{SM}}$}

Figures 3(a) to 3(d) show the constraints arising from $a_{\mu}^{\text {SUSY }}$ when the present experimental data from Brookhaven is compared with the standard model result. Here we consider the residual amount $a_{\mu}^{\mathrm{expt}}-a_{\mu}^{\mathrm{SM}}$ to limit $a_{\mu}^{\mathrm{SUSY}}$ within the $2 \sigma$ level of combined error estimates, added in quadratures $\left(-43.0 \times 10^{-10}<a_{\mu}^{\text {SUSY }}<142.8 \times 10^{-10}\right)$. Considering the largest possible $\left|a_{\mu}^{\mathrm{SUSY}}\right|$ within the model, we see that, essentially, a constraint exists only for $\mu<0$. The regions excluded by $a_{\mu}^{\text {SUSY }}$ bounds when combined with the disallowed regions (labeled by $X$ ) characteristic of the minimal AMSB model itself, along with the experimental constraints on various sparticle masses, a value of $m_{0}$ below $275 \mathrm{GeV}$ is completely eliminated for any value of $m_{3 / 2}$ [see Fig. 3(a)]. The nature of the excluded region as marked by $X$ principally originates from sleptons turning into the LSP and then becoming tachyonic at the electroweak scale (see Sec. V). The same for $\tan \beta=40$ as shown in Fig. 3(c) amounts to $m_{0}$ $\sim 375 \mathrm{GeV}$. Additionally, as seen in the same displays, significantly larger $m_{0}$ values than what are mentioned above are excluded for a limited region of $m_{3 / 2}$.

Constraining the minimal AMSB model via $a_{\mu}^{\text {SUSY }}$ can be further effective in the $\left(m_{\tilde{\chi}_{1}^{ \pm}}, m_{\tilde{\nu}_{\mu}}\right)$ plane [see Figs. 3(b) and $3(\mathrm{~d})$ ] because, as noted earlier, the chirality nondiagonal lighter chargino terms dominate over the other contributions. A value of $m_{\tilde{\nu}_{\mu}}$ below 225 (325) GeV for $\tan \beta=25$ (40) is explicitly ruled out via the current limit on $a_{\mu}^{\text {SUSY }}$. Here we note that in situations similar to the minimal AMSB model, 

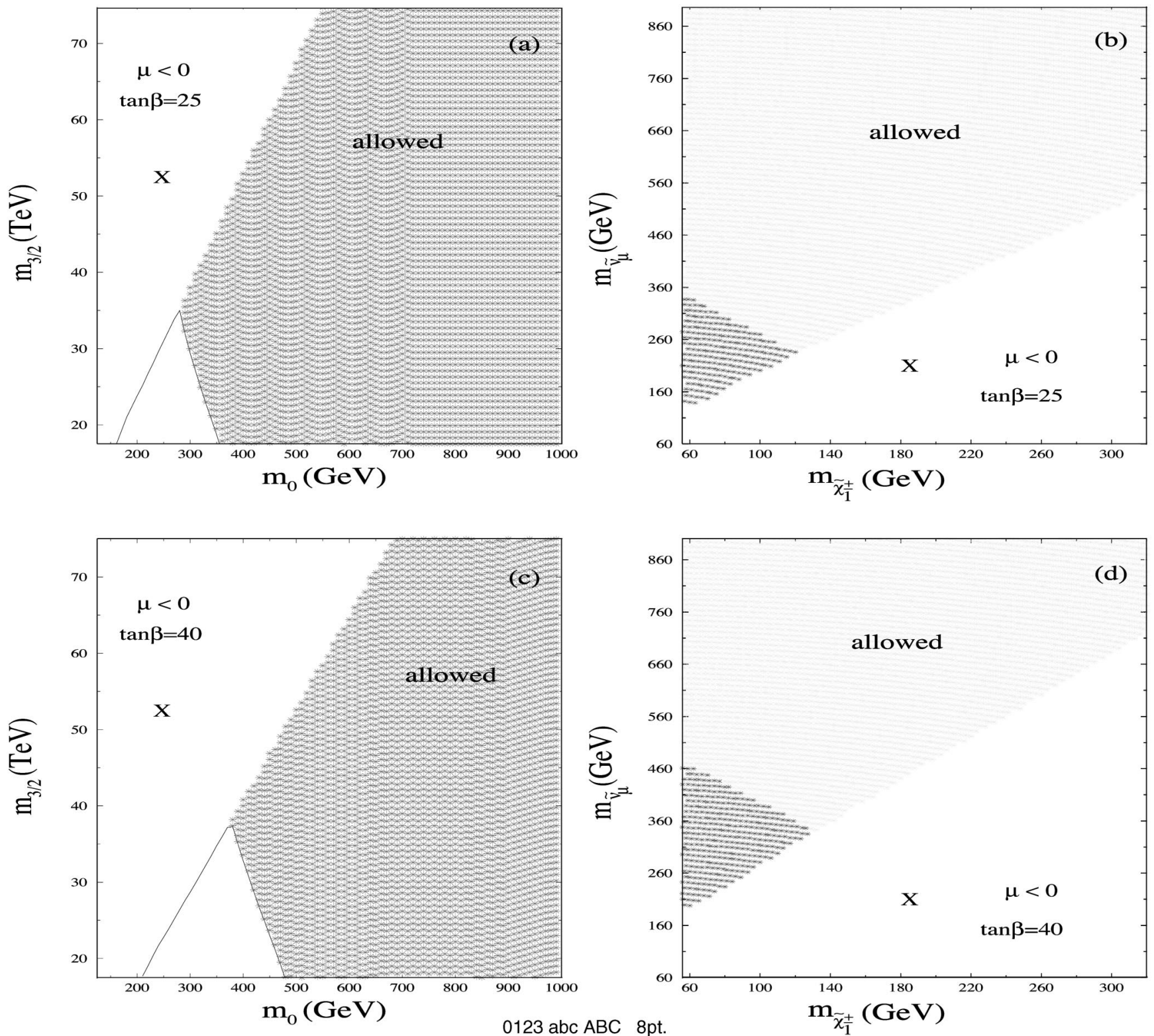

FIG. 3. Constraints in the $\left(m_{0}-m_{3 / 2}\right)$ and $\left(m_{\tilde{\chi}_{1}^{ \pm}-m_{\nu_{\mu}}}\right)$ planes from the present limits of $a_{\mu}^{\text {SUSY }}$ for $\mu<0$. Regions allowed by the $a_{\mu}^{\text {SUSY }}$ constraint are labeled as allowed. Regions marked with $X$ are generally disallowed zones for the SUSY spectra within minimal AMSB. Small white triangular regions below the allowed areas in the left hand side figures are disallowed via the $a_{\mu}^{\text {SUSY }}$ limit. The same regions in the right hand side are darkly shaded.

where $m_{\chi_{1}^{ \pm}}$and $m \tilde{\chi}_{1}^{0}$ masses are almost degenerate and sneutrinos are light, the present experimental lower bound of $m_{\chi_{1}^{ \pm}}^{ \pm}$ is $56 \mathrm{GeV}$ [33]. The white regions denoted by $X$ in the bottom of the $a_{\mu}^{\text {SUSY }}$ allowed and disallowed zones of Figs. 3(b) and 3(d) are disallowed for the same reasons as mentioned before and we will further comment on them in Sec. V while discussing similar regions in Figs. 4 and 5.

\section{B. Probing the minimal AMSB scenario further via $a_{\mu}^{\text {SUSY }}$ and} the BNL experiment at its predicted level of accuracy

The uncertainty level of $\delta a_{\mu}^{B N L}=4 \times 10^{-10}$, which is going to be achieved at Brookhaven within a few years, will, at least, significantly constrain the parameter space of a theory beyond the standard model. Considering this predicted level of accuracy, we constrain $a_{\mu}^{\text {SUSY }}$ within the $2 \sigma$ limit (see Figs. 4 and 5), where $\sigma$ is obtained from the predicted uncertainty level of BNL experiment and the error associated with the SM result, added in quadratures. The assumed nondiffering central estimates of the experimental and theoretical results would be the limiting scenario of seeing no deviation from the standard model result. This analysis would be valid in the situation when the experiment is complete and no deviation from the standard model is seen within the error limits. This is in a similar line to what has been seen in Refs. $[5,34]$ for supersymmetric as well as nonsupersymmetric 

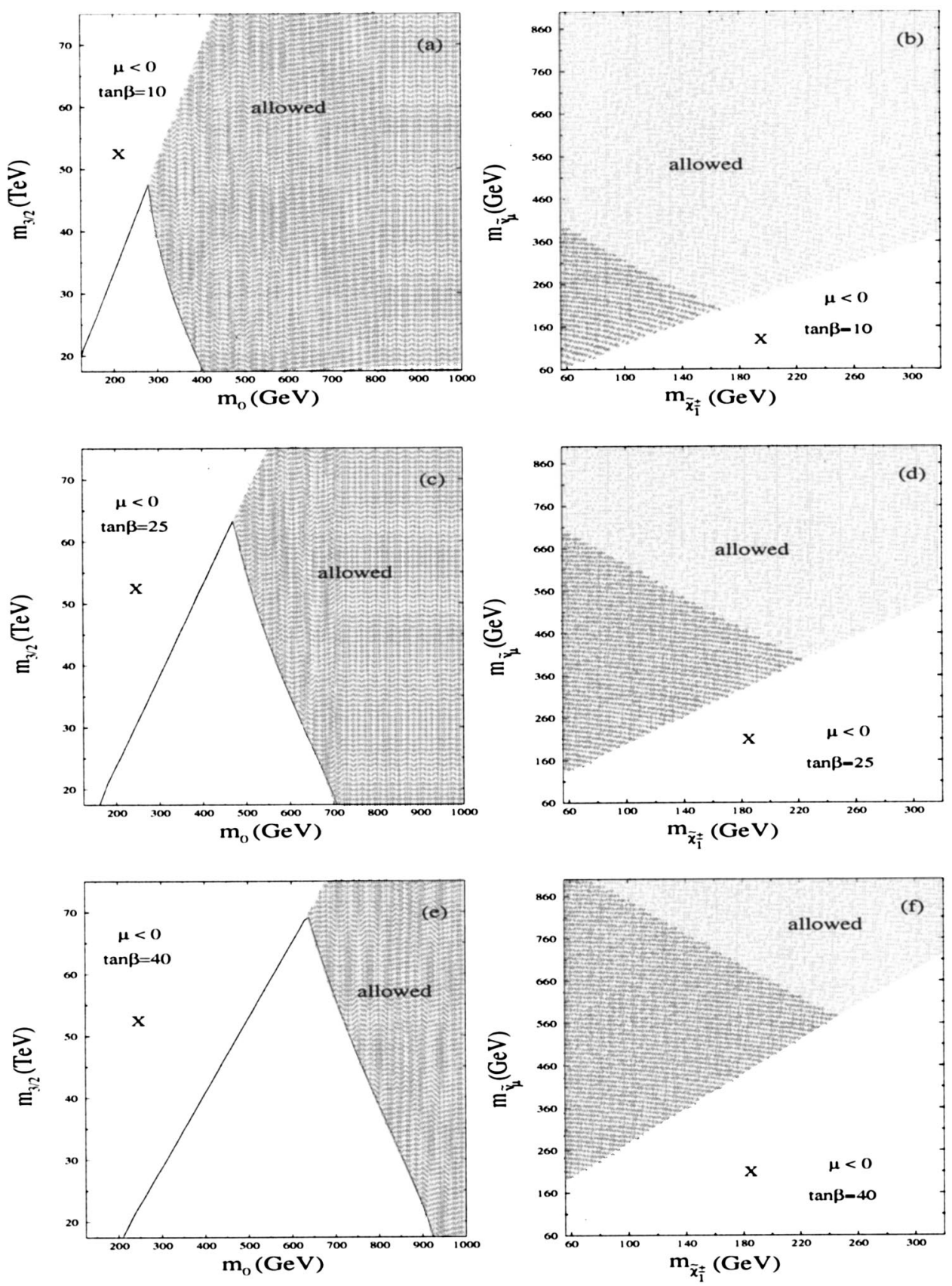

FIG. 4. Constraints in the $\left(m_{0}-m_{3 / 2}\right)$ and $\left(m_{\tilde{\chi}_{1}^{ \pm}-m_{\nu_{\mu}}}\right)$ planes within the no deviation from SM scenario of $a_{\mu}^{\text {SUSY }}$ as explained in the text for $\mu<0$. Regions allowed by the $a_{\mu}^{\mathrm{SUSY}}$ constraint are labeled as allowed. Regions marked with $X$ are generally disallowed zones for the SUSY spectra within minimal AMSB. White triangular regions below the allowed areas in the left hand side figures are disallowed via the $a_{\mu}^{\text {SUSY }}$ limit. The same regions in the right hand side are darkly shaded.

theories. On a further note, we assume that the hadronic error in $a_{\mu}^{\text {had } 1}$ would be staying at its present level. A reduction, on the other hand, which will occur in the near future, would further constrain a similar analysis.

The lower triangular regions in the $\left(m_{3 / 2}, m_{0}\right)$ plane of Figs. 4 and 5 are the disallowed zones, where $\left|a_{\mu}^{\mathrm{SUSY}}\right|$ exceeds the $2 \sigma$ limit of the combined uncertainty. The same result within the $a_{\mu}^{\text {SUSY }}$-relevant mass pairs $\left(m_{\tilde{\chi}_{1}^{ \pm}}, m_{\tilde{v}_{\mu}}\right)$ is presented in the right hand sides of Figs. 4 and 5. We note that, corresponding to $\tan \beta=10$ and 25, the minimal AMSB satisfied parameter space is reasonably identical, with respect to the sign of $\mu$. In Figs. 4(e) and 5(e), however, the regions differ in this aspect, and we will come back to them in Sec. $\mathrm{V}$.

Figures 4 and 5 indicate that $m_{0}<275$ (475) GeV domains will be entirely eliminated for $\tan \beta=10$ (25) for both signs of $\mu$. For $\tan \beta=40$, the corresponding limits are 625 $\mathrm{GeV}$ for $\mu<0$ and $800 \mathrm{GeV}$ for $\mu>0$. The limit of $m_{0}$ $\leqslant 800 \mathrm{GeV}$ for $\mu>0$ appears because of reasons which we will discuss soon. Within the $\left(m_{\tilde{\chi}_{1}^{ \pm}}, m_{\tilde{\nu}_{\mu}}\right)$ planes of the right 

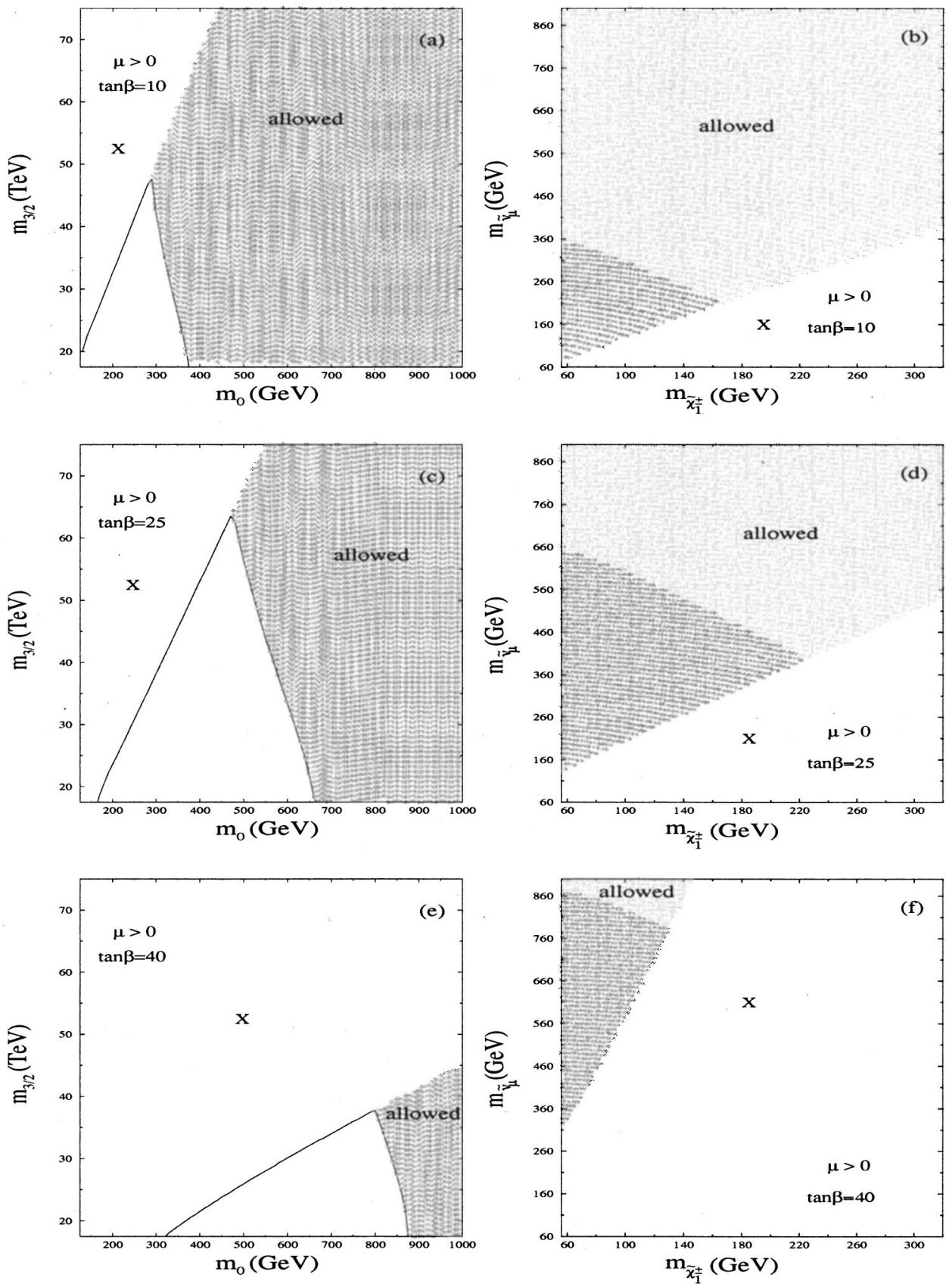

FIG. 5. Constraints in the $\left(m_{0}-m_{3 / 2}\right)$ and $\left(m_{\tilde{\chi}_{1}^{ \pm}-m_{\nu_{\mu}}}\right)$ planes within the no deviation from SM scenario of $a_{\mu}^{\text {SUSY }}$, as explained in the text for $\mu>0$. Regions allowed by the $a_{\mu}^{\mathrm{SUSY}}$ constraint are labeled as allowed. Regions marked with $X$ are generally disallowed zones for the SUSY spectra within minimal AMSB. White triangular regions below the allowed areas in the left hand side figures are disallowed via the

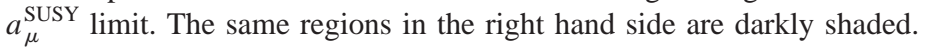

hand side of Figs. 4 and 5, we find that for $\mu<0$ and $\tan \beta$ $=10,25$, and 40, values of $m_{\tilde{v}_{\mu}}$ less than 210, 400, and 560 $\mathrm{GeV}$ are excluded. The situation for $\mu>0$ is identical for $\tan \beta=10$ and 25. A significant difference between the signs of $\mu$ can be seen now, switching to $\mu>0$ and $\tan \beta=40$. A similar disallowed range will be very stringent here; namely, $m_{\tilde{\nu}_{\mu}}$ below $780 \mathrm{GeV}$ will be excluded.

Interestingly, an important result is found when this analysis of $a_{\mu}^{\text {SUSY }}$ is combined with the $b \rightarrow s+\gamma$ constraint.
The constraint from $b \rightarrow s+\gamma$ within the minimal AMSB scenario as analyzed in Ref. [13] is somewhat complementary to what we find here from $a_{\mu}^{\mathrm{SUSY}}$. This is because the $b \rightarrow s+\gamma$ calculation, which has many special features in AMSB models, puts severe mass limits for $\mu>0$ and much smaller limits for $\mu<0$. On the other hand, within the above scenario of seeing no deviation from the SM result once the experiment is performed at the predicted level of accuracy, $a_{\mu}^{\text {SUSY }}$ limits in the minimal AMSB model impose a very significant constraint for both $\mu<0$ and $\mu>0$ cases. 


\section{GENERALLY DISALLOWED PARAMETER ZONES}

A discussion about the generally eliminated parameter space may be useful in studying the supersymmetric contribution to muon $(g-2)$ in a given SUSY model, because a combined constraint from $a_{\mu}^{\text {SUSY }}$, as well as from any generic disallowedness, results in simpler and definite predictions. Restricting the stau from becoming tachyonic corresponds to a significant constraint in AMSB models. In this section, by allowedness we mean valid input parameters from the model, in addition to satisfying various experimental lower bounds of sparticle masses, without reference to any $a_{\mu}^{\text {SUSY }}$ constraint.

We will first describe a few observations as revealed from our numerical analysis. For a given $m_{0}$, the larger $m_{3 / 2}$ values falling within the region labeled by $X$ in Figs. 4 and 5 (also in Fig. 3) are eliminated because of a decreasing stau mass $\left(m_{\tau_{1}}\right)$, which either goes below the experimental lower limit of $70 \mathrm{GeV}$ [35] or becomes the LSP, hence discarded in our $R$-parity conserved scenario. Thereafter, with a further increase of $m_{3 / 2}$, the stau becomes tachyonic. We also see that the maximum possible $m_{3 / 2}$ for a given $m_{0}$, as allowed by the minimal AMSB model, is larger for a smaller $\tan \beta$. Thus, for $\mu<0$ and $m_{0}=400 \mathrm{GeV}$, comparing Figs. 4(a), 4(c), and 4(e) we find that such maximum possible values of $m_{3 / 2}$ are approximately 67,52 , and $41 \mathrm{TeV}$ for $\tan \beta=10$, 25 , and 40, respectively. On the other hand, for a given $\tan \beta$, an allowed $m_{3 / 2}$ increases for an increase in $m_{0}$. Besides, smaller $m_{3 / 2}$ regions below the origins of the displays are eliminated via the experimental constraint of $m_{\tilde{\chi}_{1}^{ \pm}}$ $\gtrsim 56 \mathrm{GeV}$ [33].

We will try to explain, qualitatively, the behavior of stau mass with variations of the basic parameters of the model. The effect of $\tilde{\tau}_{1}$ becoming tachyonic, as described above, is best explained via $\tilde{m}_{L}^{2}$ [see Eq. (5) for $i \equiv L$ ] assuming smaller left-right slepton mixing for convenience. There are two effects in $\tilde{m}_{L}^{2}$ due to a change in $\tan \beta$, which may support or oppose each other. The first one arises from the scale invariant part of $\tilde{m}_{L}^{2}$ and the other one originates from $\mathrm{RG}$ evolution [28] of the same.

The Yukawa term in the scale invariant part of $\tilde{m}_{L}^{2}$ in Eq. (5) is intrinsically negative, itself being also gauge coupling dominated within the corresponding $\hat{\beta}$ function, for the range of $\tan \beta$ considered in this analysis. Hence, the value of $\tilde{m}_{L}^{2}$ at $M_{G}$ decreases if $\tan \beta$ is larger. We consider here moderate values of $m_{0}$ for a simpler discussion. Until $\tan \beta$ is in a smaller domain, so that $\tau$-Yukawa coupling $\left(h_{\tau}\right)$ within the scalar mass RG equation [28] may be neglected compared to the gauge terms, the RGE effect due to running from $M_{G}$ to the electroweak scale always increases $\tilde{m}_{L}^{2}$ because of gauge domination. Thus the two effects oppose each other. But within smaller $\tan \beta$ domains, regarding the value of $\tilde{m}_{L}^{2}$ at the electroweak scale for an increase in $\tan \beta$, the effect of the AMSB specified decrease in $\tilde{m}_{L}^{2}$ at $M_{G}$ is stronger than the increase due to the RGE effect. We have also verified this numerically in a broad domain of parameter ranges. As a result, $\tilde{m}_{L}^{2}$ and consequently $m_{\tau_{1}}$ decrease with an increase in $\tan \beta$. This in turn means that, for a given $m_{0}$, the upper limit of $m_{3 / 2}$ is reached sooner for a larger $\tan \beta$. This we may see from Figs. 4 and 5, as well as from the values quoted above within this section.

For a further increase in $\tan \beta(\sim 40$ in our analysis), instead of opposing, the two effects may go in the same direction, although with varying strengths, because the $\tau$-Yukawa term may now start to dominate within the RG evolution. In fact, this may also be true when $m_{0}$ is large, with $\tan \beta$ in a moderately larger domain $(\sim 25)$.

On the other hand, corresponding to the lowest $m_{3 / 2}$ values satisfying the lighter chargino experimental bound, a gradual increase of the lower limit of $m_{0}$ with an increase in $\tan \beta$ is found (see left side displays of Figs. 4 and 5), because, as explained before, the scale invariant part of $\tilde{m}_{L}^{2}$ turns further negative for increasing $\tan \beta$, and larger $m_{0}$ values are hence needed to compensate. However, there is a marked difference between the $\mu<0$ and $\mu>0$ cases for $\tan \beta=40$ [see Fig. 4(e) and 5(e)]. The upper limit of the $m_{3 / 2}$ for $\mu>0$ as allowed by the model, is much smaller compared to the same for $\mu<0$. This happens due to a generic large $\tan \beta$ effect, the effect of large SUSY-QCD loop corrections of bottom-quark mass [30]. Significantly, this correction has special features in the AMSB scenario [18], because within the same the $\mathrm{SU}(3)$ gaugino mass $\tilde{m}_{3}$ comes with a negative sign. Consequently, for $\mu>0$ and large $\tan \beta$, as a result of a large SUSY QCD loop correction, a very large $h_{b}\left(\sim h_{t}\right)$ causes $m_{H_{d}}^{2}$ for the Higgs scalar to turn sufficiently negative so that the $C P$-odd Higgs particle becomes tachyonic. However, here $\tilde{\tau}_{1}$ can still remain nontachyonic. A further increase of $m_{3 / 2}$ causes $\tilde{\tau}_{1}$ to become tachyonic, as usual.

Considering now the combined effect of the model specified disallowed space, as well as the constraint from $a_{\mu}^{\text {SUSY }}$, we find that a region below $m_{0}=800 \mathrm{GeV}$ for $\mu>0$ will be completely eliminated within the scenario of seeing no deviation from the SM. The right hand side displays of Figs. 4 and 5 also show model specified eliminated regions, as identified by $X$ in the $\left(m_{\tilde{\chi}_{1}^{ \pm}}, m_{\tilde{\nu}_{\mu}}\right)$ plane. Obviously, the masses are not independent, because they are derived from the basic set of input parameters of the minimal AMSB model. The same reason for $\tilde{\tau}_{1}$ becoming tachyonic eliminates a large region within the zones $X$. The disallowed $X$ zone is large for $\mu>0$ and $\tan \beta=40$ in Fig. 5(f), compared to the same for $\mu<0$, as shown in Fig. 4(f). This occurs because of the same reason for which the upper limit of $m_{3 / 2}$ is smaller in Fig. 5(e), which we have explained before, and due to the fact that the lighter chargino is $W$-ino dominated within AMSB, thus $m_{\chi_{1}^{ \pm}}$being almost proportional to $m_{3 / 2}$.

\section{CONCLUSION}

We have computed the supersymmetric contribution to the anomalous magnetic moment of the muon within the minimal anomaly mediated supersymmetry breaking model. 
There are one-loop contributions involving charginosneutrino and neutralino-smuon parts. The chiral interference term involving the lighter chargino is seen to contribute the most to $a_{\mu}^{\text {SUSY }}$ than the other chargino and neutralino terms, and this also results in a definite sign relationship between $a_{\mu}^{\text {SUSY }}$ and $\mu$. In addition, this also gives an almost proportional relationship of $\left|a_{\mu}^{\text {SUSY }}\right|$ with $\tan \beta$. We have also seen the effect of large smuon $L-R$ mixing which causes strong partial cancellations between the various terms of the neutralino result of $a_{\mu}^{\text {SUSY }}$. This is a significantly important result of $a_{\mu}^{\text {SUSY }}$ within minimal AMSB.

We have analyzed the constraint coming from current values of $a_{\mu}$ from the standard model and the ongoing experiment at Brookhaven, assuming that the difference appears due to SUSY. The constraint which exists only for $\mu<0$ shows that, for $\tan \beta=25$ (40), regions with $m_{0}<275$ (375) $\mathrm{GeV}$ and, correspondingly, $m_{\tilde{\nu}_{\mu}}<225$ (325) GeV are eliminated. We have also investigated the constraint from $a_{\mu}^{\text {SUSY }}$ that would result if the Brookhaven experiment with its already predicted level of accuracy finds no deviation from the standard model result. In this scenario, one finds that for $\mu$ $<0$ and $\tan \beta=10,25$, and 40, the lower bounds of $m_{0}$ would be 275,475 , and $625 \mathrm{GeV}$, while the corresponding lower limits for $m_{\tilde{\nu}_{\mu}}$ would be 210 , 400, and $560 \mathrm{GeV}$, respectively. The lower bounds for $\mu>0$ are identical to $\mu$ $<0$, except for $\tan \beta=40$, where $m_{0}<800 \mathrm{GeV}$ and correspondingly $m_{\tilde{\nu}_{\mu}}<780 \mathrm{GeV}$ regions would be excluded. This happens due to a large SUSY-QCD correction to the bottomquark mass, a large $\tan \beta$ effect.

We have also compared our constraint with the same obtained from $b \rightarrow s+\gamma$ from Ref. [13]. We found that the high accuracy level of the BNL experiment will be very useful to constrain the model for $\mu<0$, because the $b \rightarrow s+\gamma$ limit is effective only for $\mu>0$. Furthermore, we have also analyzed the generically disallowed zones within the parameter space of the model, because a combined constraint from $a_{\mu}^{\text {SUSY }}$ and such invalid parameter ranges lead to a stronger prediction.

\section{ACKNOWLEDGMENTS}

D.K.G. wishes to acknowledge the hospitality provided by the Theory Division, CERN, where part of this work was completed.
[1] B. L. Roberts, in Proceedings of the XIX International Symposium on Lepton and Photon Interactions at High Energy, Stanford University, 1999, hep-ex/0002005; R. M. Carey et al., Phys. Rev. Lett. 82, 1632 (1999); D. Hertzog et al., in Proceedings of the Sixth International Symposium on Particles, Strings and Cosmology (PASCOS 98), Northeastern University, Boston, 1998, edited by P. Nath (World Scientific, Singapore, 1998); URL: http://www.g2muon.bnl.gov and http://www.phy.bnl.gov/g2muon/home.html.

[2] J. Bailey et al., Phys. Lett. 68B, 191 (1977); Nucl. Phys. B150, 1 (1979).

[3] T. Ibrahim and P. Nath, Phys. Rev. D 62, 015004 (2000).

[4] G. Cho, K. Hagiwara, and M. Hayakawa, Phys. Lett. B 478, 231 (2000); T. Blažek, hep-ph/9912460; T. Moroi, Phys. Rev. D 53, 6565 (1996); 56, 4424(E) (1997); M. Carena, G. F. Giudice, and C. E. M. Wagner, Phys. Lett. B 390, 234 (1997).

[5] U. Chattopadhyay and P. Nath, Phys. Rev. D 53, 1648 (1996).

[6] T. Goto, Y. Okada, and Y. Shimizu, hep-ph/9908499; T. Ibrahim and P. Nath, Phys. Rev. D 61, 095008 (2000); J. L. Lopez, D. V. Nanopoulos, and X. Wang, ibid. 49, 366 (1994) and references therein; T. C. Yuan, R. Arnowitt, A. H. Chamseddine, and P. Nath, Z. Phys. C 26, 407 (1984).

[7] K. T. Mahanthappa and S. Oh, in Proceedings of the International Europhysics Conference on High Energy Physics, Tampere, Finland, 1999, hep-ph/9909410; Carena et al., Ref. [4].

[8] P. Nath, R. Arnowitt, and A.H. Chamseddine, Applied $N=1$ Supergravity, ICTP Lecture Series, Vol. I (World Scientific, Singapore, 1984); H. P. Nilles, Phys. Rep. 110, 1 (1984).

[9] G. F. Giudice and R. Rattazzi, Phys. Rep. 322, 419 (1999); 322, 501 (1999).

[10] L. Randall and R. Sundrum, Nucl. Phys. B557, 79 (1999).

[11] G. F. Giudice, M. A. Luty, H. Murayama, and R. Rattazzi, J.
High Energy Phys. 12, 027 (1998).

[12] J. A. Bagger, T. Moroi, and E. Poppitz, J. High Energy Phys. 04, 009 (2000).

[13] J. L. Feng and T. Moroi, Phys. Rev. D 61, 095004 (2000).

[14] T. Gherghetta, G. F. Giudice, and J. D. Wells, Nucl. Phys. B559, 27 (1999).

[15] S. Su, Nucl. Phys. B573, 87 (2000).

[16] J. L. Feng, T. Moroi, L. Randall, M. Strassler, and S. Su, Phys. Rev. Lett. 83, 1731 (1999); F. Paige and J. Wells, hep-ph/0001249.

[17] D. K. Ghosh, P. Roy, and S. Roy, J. High Energy Phys. 08, 031 (2000). For other interesting collider aspects within minimal AMSB see Refs. [14,16].

[18] G. D. Kribs, Phys. Rev. D 62, 015008 (2000).

[19] A. Czarnecki and W. J. Marciano, invited talk at the 5th International Workshop on Tau Lepton Physics (Tau'98), Santander, Spain, 1998, hep-ph/9810512; T. Kinoshita, Phys. Rev. D 47, 5013 (1993).

[20] M. Davier and A. Höcker, Phys. Lett. B 435, 427 (1998).

[21] B. Krause, Phys. Lett. B 390, 392 (1997).

[22] M. Hayakawa and T. Kinoshita, Phys. Rev. D 57, 465 (1998). See also an alternative calculation with somewhat different underlying assumptions resulting in a larger error estimate in J. Bijnens, E. Pallante, and J. Prades, Phys. Rev. Lett. 75, 1447 (1995); 75, 3781(E) (1995); Nucl. Phys. B474, 379 (1996).

[23] T. Kinoshita and W. J. Marciano, in Quantum Electrodynamics, edited by T. Kinoshita (World Scientific, Singapore, 1990), pp. 419-478; T. Kinoshita, B. Nizic, and Y. Okamoto, Phys. Rev. D 31, 2108 (1985); T. Kinoshita, Z. Phys. C 56, S80 (1992).

[24] Z. T. Zhao and S. H. Wang, in Proceedings of the First Asian Particle Accelerator Conference (APAC 98), Tsukuba, Japan, 
1998; G. Pancheri, Acta Phys. Pol. B 30, 2243 (1999); “'Status of Experiments and Recent Results from CMD-2 Detector at VEPP-2M,' Third Workshop on Physics and Detectors for DAPHNE (DAPHNE 99), Frascati, Italy, 1999, hep-ex/ 0001049.

[25] F. Jegerlehner, in Proceedings of the IVth International Symposium on Radiative Corrections, Barcelona, 1998, hep-ph/9901386.

[26] A. Pomarol and R. Rattazzi, J. High Engery Phys. 05, 013 (1999); E. Katz, Y. Shadmi, and Y. Shirman, ibid. 08, 015 (1999); Z. Chacko, M. A. Luty, I. Maksymyk, and E. Ponton, ibid. 04, 001 (2000); R. Rattazzi, A. Strumia, and J. D. Wells, Nucl. Phys. B576, 3 (2000); I. Jack and D. R. T. Jones, Phys. Lett. B 482, 167 (2000); B. C. Allanach and A. Dedes, J. High Energy Phys. 06, 017 (2000); M. Carena, K. Huitu, and T. Kobayashi, hep-ph/0003187.

[27] J. L. Feng, K. Matchev, and T. Moroi, Phys. Rev. D 61, 075005 (2000).

[28] S. P. Martin and M. T. Vaughn, Phys. Rev. D 50, 2282 (1994).
[29] R. Arnowitt and P. Nath, Phys. Rev. D 46, 3981 (1992); V. Barger, M. S. Berger, and P. Ohmann, ibid. 49, 4908 (1994).

[30] L. J. Hall, R. Rattazzi, and U. Sarid, Phys. Rev. D 50, 7048 (1994); R. Hempfling, ibid. 49, 6168 (1994); M. Carena, M. Olechowski, S. Pokorski, and C. Wagner, Nucl. Phys. B426, 269 (1994); D. Pierce, J. Bagger, K. Matchev, and R. Zhang, ibid. B491, 3 (1997).

[31] H. Baer, M. Díaz, P. Quintana, and X. Tata, J. High Energy Phys. 04, 016 (2000).

[32] For naturalness in MSUGRA, see Ref. [27] and K. L. Chan, U. Chattopadhyay, and P. Nath, Phys. Rev. D 58, 096004 (1998) and references therein. For naturalness within minimal AMSB, see Ref. [13].

[33] M. Maltoni, hep-ph/0003315.

[34] T. Blažek [4]. For an analysis using the two-Higgs-doublet model see M. Krawczyk and J. Zochowski, Phys. Rev. D 55, 6968 (1997).

[35] LEP SUSY Working Group, ALEPH, DELPHI, L3, OPAL experiments, note No. LEPSUSYWG/99-01.1. 